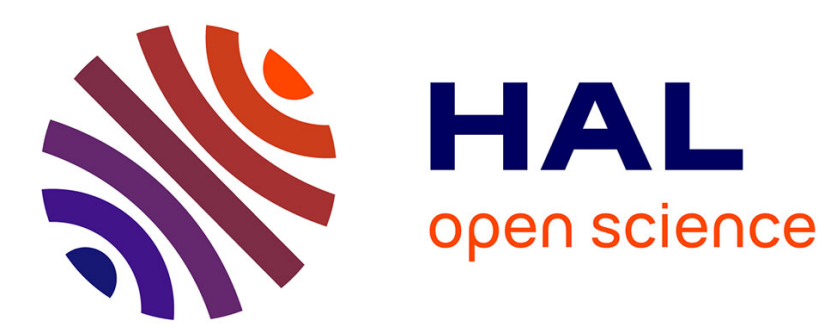

\title{
Time scaling of the ageing process in spin-glasses: a study in CsNiFeF 6
}

M. Ocio, M. Alba, J. Hammann

\section{To cite this version:}

M. Ocio, M. Alba, J. Hammann. Time scaling of the ageing process in spin-glasses: a study in CsNiFeF 6. Journal de Physique Lettres, 1985, 46 (23), pp.1101-1107. 10.1051/jphyslet:0198500460230110100 . jpa-00232943

\section{HAL Id: jpa-00232943 https://hal.science/jpa-00232943}

Submitted on 1 Jan 1985

HAL is a multi-disciplinary open access archive for the deposit and dissemination of scientific research documents, whether they are published or not. The documents may come from teaching and research institutions in France or abroad, or from public or private research centers.
L'archive ouverte pluridisciplinaire HAL, est destinée au dépôt et à la diffusion de documents scientifiques de niveau recherche, publiés ou non, émanant des établissements d'enseignement et de recherche français ou étrangers, des laboratoires publics ou privés. 
Classification

Physics Abstracts

$75.50-75.90$

\title{
Time scaling of the ageing process in spin-glasses : a study in $\mathrm{CsNiFeF}_{6}$
}

\author{
M. Ocio, M. Alba and J. Hammann \\ Service de Physique du Solide et de Résonance Magnétique, \\ CEN-Saclay, 91191 Gif-sur-Yvette Cedex, France
}

(Reçu le 11 juillet 1985, accepté le 8 octobre 1985)

\begin{abstract}
Résumé. - Les propriétés de vieillissement de la décroissance de l'aimantation thermorémanente (TRM) en fonction du temps dans $\mathrm{CsNiFeF}_{6}$ sont analysées à partir d'une théorie phénoménologique d'échelle déjà utilisée dans le cas des propriétés mécaniques des polymères vitreux. Nous en déduisons, pour la décroissance de la TRM, une loi donnée par le produit d'une exponentielle étirée par une loi de puissance.
\end{abstract}

\begin{abstract}
The ageing properties of the time decay of the thermoremanent magnetization (TRM) in $\mathrm{CsNiFeF}_{6}$ are analysed on the basis of a phenomenological theory of time scaling as yet used in the case of the mechanical properties of glassy polymers. We find that the time decay law takes the form of the product of a stretched exponential by a power law.
\end{abstract}

Ageing in spin glasses is a field of growing interest. It is now well known that at low temperatures, many properties are time dependent, showing that in the time scale of laboratory experiments, the spin-glass has not reached its equilibrium state. Recent experiments on the time variation of the zero field cooled magnetization $[1,2]$ as well as of the thermoremanent magnetization (TRM) [3, 4] in metallic spin-glasses emphasize the influence of the time the material has been kept below the glass temperature $T_{\mathrm{g}}$ before any field variation (waiting time). It has been already quoted that relaxation phenomena in spin glasses present some degree of resemblance with relaxation in ordinary glasses and glassy polymers [2, 5]. Ageing is a general property of the glassy state, and is found in all glasses, irrespective of their chemical nature. Furthermore, it appears that all glassy polymers age in a similar way [6]. Extensive studies of small strain creep in numerous classes of polymeric glasses as well as amorphous compounds show that the detailed physical and chemical structure of the material is of no direct importance. It has been noted, that materials whose creep curves show stretched exponential behaviour, always yield ageing effects [6]. Recent experimental results [3,4] as well as theoretical approaches [7] support the applicability of such a relaxation law in spin glasses. Although the relation between non classical relaxation and ageing is not understood at the day, the present work is an attempt to apply in spin glasses the concepts used to analyse ageing in glassy materials. The material studied is $\mathrm{CsNiFeF}_{6}$, a non dilute crystalline compound of the modified pyrochlore structure. In this compound, topological frustation arises from next nearest antiferromagnetic interactions, and disorder is generated from the random distribution of $\mathrm{Ni}^{2+}$ and $\mathrm{Fe}^{3+}$ on the same magnetic lattice. The freezing temperature is $5.4 \mathrm{~K}$ [8]. 


\section{Experimental details and results.}

We have measured the time decay of the thermoremanent magnetization $\sigma$ of a powdered sample of $\mathrm{CsNiFeF}_{6}$ with dimensions $\varnothing=6 \mathrm{~mm}$ and $h=15 \mathrm{~mm}$. To achieve a good thermalization of the powder, the latter was embedded in silicon grease (about $40 \%$ in vol) and packed in a copper sample holder, longitudinally slit to avoid eddy currents. The sample was placed in one of the coils of a first order gradiometer connected to a LETI M.S. 03 SQUID system. The measuring procedure was the following :

1) a magnetic field $(H=15 \mathrm{mOe})$ was applied to the sample maintained at $6.5 \mathrm{~K}\left(T / T_{\mathrm{g}}=1.2\right)$ in the paramagnetic regime;

2) then the sample was cooled at a constant rate to a temperature $T$ in the spin glass region, $t_{\mathrm{c}}$ being the cooling time elapsed between $T_{\mathrm{g}}$ and $T$;

3) after a certain waiting time $t_{\mathrm{w}}$ at temperature $T$, the magnetic field was switched off and the time decay of the TRM was recorded as a function of the observation time $t$ over a period up to 24 hours;

4) next, the sample was warmed up to $6.5 \mathrm{~K}$ and the signal recorded in order to check the baseline. The measurements were performed at $3.2 \mathrm{~K}, 4.2 \mathrm{~K}, 4.5 \mathrm{~K}$ and $4.7 \mathrm{~K}$ for cooling times between $150 \mathrm{~s}$ and $250 \mathrm{~s}$ according to the final temperature $T$, and for waiting times of about $100 \mathrm{~s}, 1000 \mathrm{~s}, 10000 \mathrm{~s}$ and $86000 \mathrm{~s}$.

Figures 1 and 2 are log-log plots of the time decay of the TRM versus $t$, for the four waiting times at each investigated temperature. Inspection of figure 1 shows that it is not possible to describe the entire decay curves by means of any kind of simple law, i.e. logarithm of time, power law of time, or even stretched exponential of time. We observe that there is a time dependence of the FC magnetization during $t_{\mathrm{w}}$, and that the initial value of $\sigma$ after the field is turned off, depends on $t_{\mathrm{w}}$.

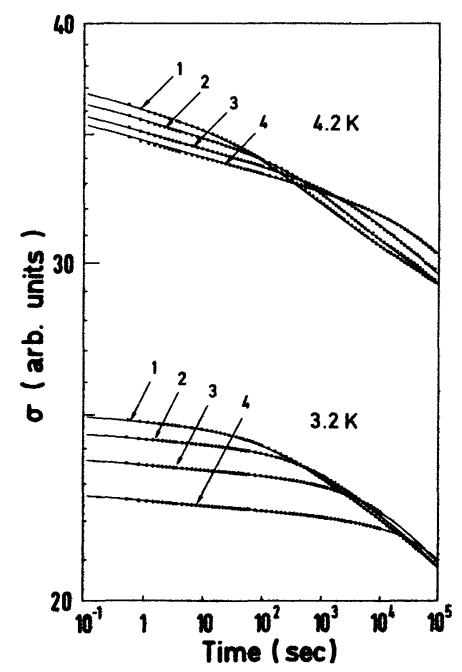

Fig. 1. - TRM decay of $\mathrm{CsNiFeF}_{6}$ at $3.2 \mathrm{~K}$ and $4.2 \mathrm{~K}$. The waiting times are $1: 140 \mathrm{~s} ; 2: 10^{3} \mathrm{~s} ; 3: 10^{4} \mathrm{~s}$; $4: 8.6 \times 10^{4} \mathrm{~s}$. Full lines are calculated values (see text). 


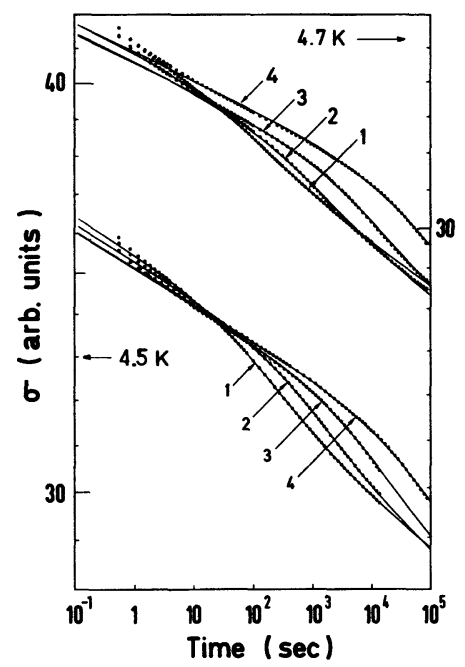

Fig. 2. - TRM decay of $\mathrm{CsNiFeF}_{6}$ at $4.5 \mathrm{~K}$ and $4.7 \mathrm{~K}$. Arrows refer to the vertical axes for each group of curves. Waiting times are given in figure 1. Full lines are calculated values (see text).

\section{Time scaling.}

Examination of the results at $3.2 \mathrm{~K}$ and $4.2 \mathrm{~K}$ shows that, in the " momentary " part of the decay (for $t<t_{\mathrm{w}}$ ) the curves can be superposed by shifts in the log-log plot, the horizontal component of the shift being slightly smaller than the logarithm of the ratio of the waiting times. Lundgren et al. [1] were the first to report the effect of $t_{\mathrm{w}}$ on the dynamical properties of spin glasses. In our results on $\mathrm{CsNiFeF}_{6}$ the shifting properties of the " momentary part » of the decay curves at $3.2 \mathrm{~K}$ and $4.2 \mathrm{~K}$, and their shape at long observation time, present rather close similarities with the creep properties of glassy materials. In an exhaustive analysis of the ageing of amorphous polymers and other materials, Struik [6] derived a phenomenological theory of ageing, which can account very well for the ageing of the TRM in $\mathrm{CsNiFeF}_{6}$, reported here. The shifting property between two curves at $t_{\mathrm{w} 1}$ and $t_{\mathrm{w} 2}>t_{\mathrm{w} 1}$ can be formulated as

$$
\sigma_{t_{\mathrm{w} 1}}(t)=D \sigma_{t_{\mathrm{w} 2}}(a t)
$$

where $D$ is the ratio between initial values of $\sigma_{t_{\mathrm{w} 2}}$ and $\sigma_{t_{\mathrm{w} 1}}$. The " acceleration factor " $a$ is a function of $t_{\mathrm{w} 2} / t_{\mathrm{w} 1}$. The simplest choice for $a$ is $\log a=\mu \log \left(t_{\mathrm{w} 2} / t_{\mathrm{w} 1}\right)$, i.e. $a=\left(t_{\mathrm{w} 2} / t_{\mathrm{w} 1}\right)^{\mu}$ where $\mu$ is a constant with value $\mu \leqslant 1$ in the case of strongly self retarded processes. The meaning of $a$ is that between $t_{\mathrm{w} 1}$ and $t_{\mathrm{w} 2}$ all the characteristic times of the relaxation spectrum are multiplied by a factor $a$.

Now consider the curve measured after a waiting time $t_{\mathrm{w}}$, and for $t>t_{\mathrm{w}}$. The total ageing time is $t_{\mathrm{w}}+t$, so $a(t)=\left(t_{\mathrm{w}} / t_{\mathrm{w}}+t\right)^{\mu}$. Thus, in the interval between $t$ and $t+\mathrm{d} t$, relaxation is $a$ times slower than it would have been if the material had kept its $t=0$ properties. The time interval $\mathrm{d} t$ is equivalent to an effective time interval $\mathrm{d} \lambda$ given by $\mathrm{d} \lambda=a(t) \mathrm{d} t$. Therefore $\lambda(t)=\int_{0}^{t} a(\tau) \mathrm{d} \tau$, and, from the expression of $a, \lambda$ is given by

$$
\lambda=\frac{t_{\mathrm{w}}}{1-\mu}\left[\left(1+\frac{t}{t_{\mathrm{w}}}\right)^{1-\mu}-1\right]
$$


for the waiting time $t_{\mathrm{w}}$. Then, the « constant age decay » $\overline{\sigma_{t_{\mathrm{w}}}}$ can be derived by

$$
\overline{\sigma_{t_{\mathrm{w}}}}(\lambda)=\sigma_{t_{\mathrm{w}}}(t) \text {. }
$$

The « momentary » curve is simply the common part of $\overline{\sigma_{t_{\mathrm{w}}}}(\lambda)$ and $\sigma_{t_{\mathrm{w}}}(t)$ for $t<t_{\mathrm{w}}$, where $t=\lambda$. Finally, a "master curve » can be constructed by renormalizing all the "constant age » curves to a standard waiting time $t_{\mathrm{w} 0}$, by a transformation to reduced effective time $\xi$

$$
\xi=\lambda / a t_{\mathrm{w} 0}=\lambda / t_{\mathrm{w}}\left(t_{\mathrm{w}} / t_{\mathrm{w} 0}\right)^{\mu-1} .
$$

The above transformation has been performed in our TRM curves for $3.2 \mathrm{~K}$ and $4.2 \mathrm{~K}$. Although there is no need for any adjustable parameter in the theory, a problem arose in our case from the determination of the real physical waiting time. Must the cooling time $t_{\mathrm{c}}$ be included in the waiting time ? To solve this question $\mu$ was first determined from the shifts of the three curves at long $t_{\mathrm{w}}$, for which $t_{\mathrm{c}}$ is unimportant. Then the true $t_{\mathrm{w}}$ for the other curve was determined by fitting it to the long $t_{\mathrm{w}}$ curves with the same value of $\mu$. The true waiting time appeared to be the time elapsed at temperature $T$, plus a rate between $15 \%$ and $30 \%$ of the cooling time $t_{c}$ according to the working temperature. A master curve was then obtained by plotting $\sigma / \sigma_{\text {ref }}$ (where $\sigma_{\text {ref }}$ is the value of $\sigma$ at an arbitrary given time), as a function of the reduced effective time $\xi$ calculated according to relation (2) with $t_{\mathrm{wo}}=10^{4} \mathrm{~s}$. The introduction of $\sigma_{\text {ref }}$, must be done in order to take care of the $t_{\mathrm{w}}$ dependence of the initial remanent magnetization. The shift property of the curves is less evident when the results at $4.5 \mathrm{~K}$ and especially $4.7 \mathrm{~K}$ are considered, due to the short time behaviour of the decay. Although this behaviour is not well understood up to now, it seems that it could be interpreted as the effect of a $t_{\mathrm{w}}$ independent additional relaxation. Tentatively, we have applied the same analysis, but, since the procedure used above seemed rather hazardous, $\mu$ was obtained by linear regression analysis to the best superposition of the curves in effective time.

Figure 3 is a log-log plot of the reduced TRM values, $\sigma / \sigma_{\text {ref }}$, as a function of reduced effective time $\xi$ for the four temperatures investigated; $\sigma_{\text {ref }}$ has been chosen, for each temperature, in order to separate the curves one from another. In the inset, the values of $\mu$ are plotted as a function of $T$. Figure 3 strongly supports the existence of a time scaling in the ageing properties of spin glasses, similar to that observed in the glassy polymers. However, contrary to the case of polymers [6] the " master curves » depend on the temperature.

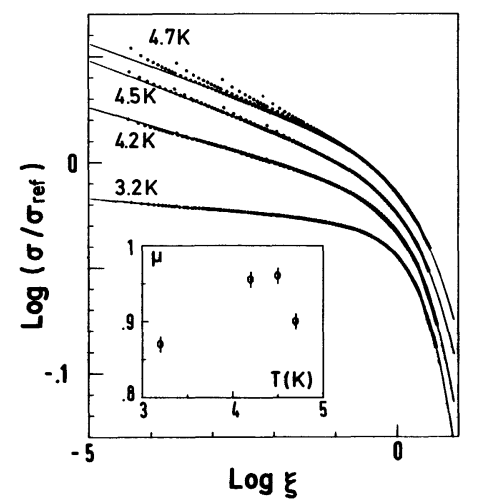

Fig. 3. - Normalized TRM decay of $\mathrm{CsNiFeF}_{6}$ as a function of reduced effective time $\xi$, and for $t_{\mathrm{w}}=10^{4} \mathrm{~s}$. Full lines are calculated values. In the inset, value of the shifting parameter $\mu$ versus temperature. 
It must be emphasized that the " master curves " would represent the transient response of the material versus $t / t_{\mathrm{w}}$, if it were possible to hold it at constant age $t_{\mathrm{w}}$, i.e. if the relaxation time distribution remained fixed at its value at $t_{\mathrm{w}}$. The correlation function describing the relaxation time distribution at $t_{\mathrm{w}}$ can thus be derived from the « master curves ».

\section{Analysis of the master curves.}

In the recent works of Chamberlin et al. [9, 3] and Hoogerbeets et al. [4], the measured « momentary » TRM decays were analysed in terms of a stretched exponential law of the general form

$$
\sigma=\sigma_{0} \exp -\left(\frac{t}{\tau_{p}}\right)^{1-n}
$$

Since Kohlrausch [10], the relaxation in many complex, strongly interacting materials was shown to follow such a time law. In the framework of glassy relaxation, several models lead to a stretched exponential time dependence [11-13].

It is true that the " momentary " part of our TRM curves could be satisfactorily accounted for by stretched exponentials with $n$ close to 1 . But this would be impossible in the long time part. On the other hand, the " master curves " as defined above, cannot be fitted by simple stretched exponentials.

It should be noted that in all the theories cited above, the stretched exponential behaviour is always multiplied by a prefactor which governs the relaxation at short times. This prefactor is generally a negative power law of time, with an exponent more or less connected to that of the stretched exponential.

Tentatively, we have fitted the master curves of figure 3 by the following law

$$
\sigma=\sigma_{0}\left(\frac{t}{\tau_{\mathrm{p}}}\right)^{-\alpha} \exp -\left(\frac{t}{\tau_{\mathrm{p}}}\right)^{1-n}
$$

which, when transposed in reduced effective units yields

with

$$
\sigma=\sigma_{0}(b \xi)^{-\alpha} \exp -(b \xi)^{1-n}
$$

$$
\tau_{\mathrm{p}}\left(t_{\mathrm{w}}\right)=\frac{t_{\mathrm{w} 0}}{b}\left(\frac{t_{\mathrm{w}}}{t_{\mathrm{w} 0}}\right)^{\mu} .
$$

The fit has been performed by non linear regression analysis. The full lines in figure 3 represent calculated values. The values of the parameters are listed in table I. Apart from the short time tails in the curves at $4.5 \mathrm{~K}$ and $4.7 \mathrm{~K}$, the deviations are less than $\pm 0.5 \%$. For $95 \%$ confidence,

Table I. - Value of the parameters used to fit the TRM decay curves as a function of reduced effective time (with $t_{\mathrm{w} 0}=10^{4} \mathrm{~s}$ ). Relations (3), (4) and (5) give the meaning of each parameter.

Parameter

$$
\begin{gathered}
\sigma_{0} \text { for } t_{\mathrm{w}}=10^{4} \mathrm{~s} \text { (volts) } \\
\alpha \\
\tau_{\mathrm{p}} \text { for } t_{\mathrm{w}}=10^{4} \mathrm{~s}(\mathrm{sec})
\end{gathered}
$$$$
n
$$

$3.2 \mathrm{~K}$

$6.7 \times 10^{5}$

0.231
$4.2 \mathrm{~K}$

30.9

0.0086

$10^{6}$

0.327
$4.5 \mathrm{~K}$

$4.7 \mathrm{~K}$ 
the parameters are defined in $\mathrm{a} \pm 1 \%$ range. The full lines in figures 1 and 2 represent the calculated values in real time. As seen in the figure, there is an excellent agreement between measured and calculated values. Recently, De Dominicis et al. [7] have derived an expression for the relaxation to equilibrium of the overlap parameter of Parisi $x$, which is also identical in form to the dependence exhibited in equation (3), apart from the fact that $\alpha=1-n$ in their model. The authors introduced the simplest choice for the relaxation rate involved in the master equation for the spin state occupancies, but they noted that the stretched exponential form seemed always present whatever the hypothesis on the relaxation rate is. It is hoped that the use of a more realistic hypothesis would lead to a decay law with a relation between $\alpha$ and $n$ in better agreement with the experiment.

A recent observation of magnetic fluctuations in $\mathrm{CsNiFeF}_{6}$ at $4.2 \mathrm{~K}$ [14] seems to be in qualitative agreement with a decay law of the form (3). For waiting times longer than $1 / 2$ hour, a stationary $1 / f$ noise spectrum is recorded in the range from $0.1 \mathrm{~Hz}$ to $100 \mathrm{~Hz}$. Below $0.1 \mathrm{~Hz}$ there is a crossover to a $1 / f^{x}$ spectrum with $1.5<x<2$. The frequency of the crossover decreases with waiting time, roughly like $1 / t_{w}$. According to linear theory of irreversible processes [15], the noise power spectrum is the Fourier transform of the correlation function, which, if time averages are equivalent to thermodynamic averages (ergodic hypothesis), is given by the transient response i.e., here, the " constant age decay ». Thus the noise at given age would be given by Fourier transforming the "master curves " at $4.2 \mathrm{~K}$. This yields a crossover from a $1 / f^{1-\alpha}$ slope $(1-\alpha \simeq 1)$ to a $1 / f^{2-n-\alpha}$ slope $(2-n-\alpha \simeq 1.7)$, at a frequency $\omega_{\mathrm{c}} \propto 1 / t_{\mathrm{w}}^{\mu}$. The meaning of this agreement is nevertheless questionable because spin glasses are strongly suspected to be non ergodic systems.

Finally, in our results, there is a discrepancy with the predictions of the models leading to stretched exponential behaviour. In the models of Ngai [11] and De Dominicis [7], the characteristic time of the stretched exponential $\tau_{\mathbf{p}}$ follows an activation law of the form

$$
\tau_{\mathrm{p}}=\tau_{0} \exp -\left(\frac{E^{*}}{T}\right)
$$

where $E^{*}$ is an effective activation energy. On the contrary, $\tau_{\mathrm{p}}$ calculated from our results in $\mathrm{CsNiFeF}_{6}$ increases with increasing temperature between $3.2 \mathrm{~K}$ and $4.7 \mathrm{~K}$. It should be noted that attempts to fit the " momentary " part of our decay curves by simple stretched exponentials in the same way as reference [4], give essentially the same result : $\tau_{\mathrm{p}}$ as well as $n$ increases with $T$ between $3.2 \mathrm{~K}$ and $4.7 \mathrm{~K}$; above $4.7 \mathrm{~K}, \tau_{\mathrm{p}}$ decreases and $n$ remains almost constant at a value close to 1 . We suggest that this unusual behaviour is due to a particular characteristic of $\mathrm{CsNiFeF}_{6}$. It has been shown [8] that in this compound, there is a large non-zero mean value of the interactions, leading to unusually large local fields.

The salient feature, nevertheless, is that the TRM decay at short times becomes faster as $T$ increases, due to the increasing $n$. When a decay law of the form (3) is used, this is taken into account by the increase of the exponent $\alpha$ of the power law, with increasing $T$.

\section{Acknowledgments.}

The authors are very indebted to $\mathrm{H}$. Bouchiat for crucial suggestions and criticisms. They thank C. Jacoboni for the preparation of the sample. 


\section{References}

[1] Lundgren, L., Nordblad, P., Svedlindh, P. and Beckman, O., J. Appl. Phys. 57 (1985) 3371.

[2] Bouchiat, H. and Mailly, D., J. Appl. Phys. 57 (1985) 3453.

[3] Chamberlin, R. V., J. Appl. Phys. 57 (1985) 3377.

[4] HoOgerbeets, R., Wei-Li Luo and Orbach, R., Phys. Rev. Lett. 55(1985) 111.

[5] Ngai, K. L., Rajagopal, A. K. and Huang, C. Y., J. Appl. Phys. 55 (1984) 1714.

[6] Struik, L. C. E., in Physical Ageing in Amorphous Polymers and Other Materials (Elsevier Scientific Publ. Co.) 1978.

[7] De Dominicis, C., Orland, H. and Lainée, F., J. Physique Lett. 46 (1985) L-463.

[8] Pappa, C., Hammann, J. and Jacoboni, C., J. Phys. C 17 (1984) 1303 and

Pappa, C., Hammann, J., Jéhanno, G. and Jacoboni, C., J. Phys. C 18 (1985) 2817.

Pappa, C., HammanN, J. and Jacoboni, C., J. Physique 46 (1985) 637.

[9] Chamberlin, C., Mozurkewich, G. and Orbach, R., Phys. Rev. Lett. 52 (1984) 867.

[10] Kohlrausch, R., Ann. Phys. (Leipzig) 12 (1847) 393.

[11] NGaI, K. L., Comments Solid State 9 (1979) 127.

[12] Grassberger, P. and Procaccia, I., J. Chem. Phys. 77 (1982) 6281.

[13] Palmer, R. G., Stein, D. L., Abrahams, E. and Anderson, P. W., Phys. Rev. Lett. 53 (1984) 958.

[14] Ocio, M., Bouchiat, H. and Monod, P., J. Physique Lett. 46 (1985) L-647 and Proceedings of ICM 85, J.M.M.M. (1986) to be published.

[15] Kubo, R., J. Phys. Soc. Jpn 12 (1957) 570. 